\title{
Ausência de influência da síndrome metabólica na atividade da artrite reumatóide
}

\author{
Absence of metabolic syndrome influence in rheumatoid arthritis activity
}

Breno Martins Farinazo ${ }^{1}$, Mauro Marcelo Furtado Real Júnior², Cezar Augusto Muniz Caldas ${ }^{3}$

Resumo Objetivo: Identificar a influência da Síndrome Metabólica (SM) na resposta ao tratamento dos pacientes portadores de Artrite Reumatoide (AR). Metodologia: Foi realizado um estudo do tipo transversal, descritivo e analítico, no período de setembro de 2013 a junho de 2014. A coleta de dados foi efetuada via protocolo de pesquisa elaborado pelos autores no ambulatório de Reumatologia do Centro de Especialidades Médicas (CEMEC) do Centro Universitário do Estado do Pará (CESUPA). Foram incluídos pacientes do sexo feminino, maiores de 18 anos e com diagnóstico de AR. Foram excluídos da pesquisa todos os pacientes tabagistas, com associação com outra doença autoimune, nefropatas, hepatopatas, mulheres gestantes, menopausadas e/ou em uso de contraceptivos hormonais. Resultados: Todas as pacientes (27) eram do sexo feminino, com média de idade de 40,89 \pm 6,71anos e de tempo de doença de 7,67 $\pm 6,02$ anos, sendo que 21 pacientes $(77,8 \%$ ) apresentaram fator reumatóide positivo. Quanto à atividade da doença, a Velocidade de Hemossedimentação (VSH) apresentou média de 35,07 $\pm 17,43 \mathrm{~mm} / \mathrm{h}$ e a Proteína C Reativa (PCR) 13,85 18,6mg/dl, respectivamente. A média do Disease Activity Score de 28 articulações (DAS28) foi de 4,69 $\pm 1,18$. A frequência de SM encontrada entre os pacientes foi de 33,3\% $(\mathrm{n}=9)$. Em relação à atividade da doença mensurada pelo DAS28 $(4,38 \pm 0,74$ vs. $4,84 \pm 1,34, \mathrm{p}=0,263)$, VHS $(33,44 \pm 13,87 \mathrm{~mm} / \mathrm{h}$ vs. $35,94 \pm 19,39 \mathrm{~mm} / \mathrm{h}, \mathrm{p}=0,709)$ e PCR $(19,33 \pm 26,66 \mathrm{mg} / \mathrm{dl}$ vs. $11,11 \pm 13,07 \mathrm{mg} / \mathrm{dl}, \mathrm{p}=0,403$ ), não houve diferença estatística entre os grupos com SM e sem SM (p=0,263). Conclusão: 0 presente estudo constatou que a presença de SM não influenciou na resposta ao tratamento nos pacientes com AR do CEMEC, não guardando relação com a atividade da doença, seja através de exames laboratoriais ou avaliada através do DAS28.

Descritores: artrite reumatóide; síndrome metabólica; inflamação.

Summary Purpose: To identify the influence of Metabolic Syndrome (MS) in the response to treatment of patients with Rheumatoid Arthritis (RA). Methods: A cross-sectional, descriptive and analytical study was carried out from September 2013 to June 2014. Data collection was done through a research protocol developed by the authors at the ambulatory of Rheumatology of the Center of Medical Specialties (CEMEC) of the University Center of the State of Pará (CESUPA). We included female patients, older than 18 years and diagnosed with RA. All the smoking patients, with association with another autoimmune disease, nephropathy, hepatopathy, pregnant women, menopausal women and / or hormonal contraceptives were excluded from the study. Results: All the patients (27) were female, with a mean age of $40.89 \pm 6.71$ years and a disease time of $7.67 \pm 6.02$ years, with 21 patients (77.8\%) presenting positive rheumatoid factor. Regarding the disease activity, the Erythrocyte Sedimentation Rate (ESR) presented a mean of $35.07 \pm 17.43 \mathrm{~mm} / \mathrm{h}$ and the C Rective Protein $13.85 \pm 18.6 \mathrm{mg} / \mathrm{dl}$, respectively. The mean Disease Activity Score of 28 joints was $4.69 \pm 1.18$. The frequency of MS found among patients was $33.3 \%(n=9)$. In relation to the disease activity measured by DAS28 (4.38 \pm 0.74 vs. $4.84 \pm 1.34, \mathrm{p}=0.263)$, HSV $(33.44 \pm 13.87 \mathrm{~mm} / \mathrm{h} v s .35 .94 \pm 19,39 \mathrm{~mm} / \mathrm{h}, \mathrm{p}=0.709)$ and CRP (19.33 $\pm 26.66 \mathrm{mg} / \mathrm{dl}$ vs. $11.11 \pm 13.07 \mathrm{mg} / \mathrm{dl}, \mathrm{p}=0.403)$, there was no statistical difference between the SM and without MS $(p=0.263)$. Conclusion: The present study found that the presence of MS did not influence the response to treatment in patients with RA of CEMEC, not either related to disease activity, through laboratory tests or evaluated through the DAS28.

Keywords: rheumatoid arthritis; metabolic syndrome; inflammation.

${ }^{1}$ Centro Universitário do Estado do Pará - CESUPA, Programa de Residência Médica em Reumatologia, Belém, PA, Brasil ${ }^{2}$ Hospital das Clínicas Gaspar Vianna, Belém, PA, Brasil

${ }^{3}$ Universidade Federal do Pará (UFPA), Belém, PA, Brasil

Fonte de financiamento: nenhuma.

Conflito de interesses: os autores declaram não haver conflitos de interesse.

Recebido: Fevereiro 21, 2018

Aceito: Julho 18, 2018

Trabalho realizado no Serviço de Reumatologia do Centro Universitário do Estado do Pará - CESUPA, Belém, PA, Brasil. 


\section{Introdução}

A Síndrome Metabólica (SM) representa uma associação de condições que têm em comum a resistência insulínica e o aumento da gordura abdominal, que se acredita estar intimamente ligada à inflamação ${ }^{1-3}$. Tal relação com a inflamação pode justificar a maior prevalência de SM em pacientes com Artrite Reumatoide (AR) e a maior atividade da doença em pacientes com AR que apresentam SM demonstrada em alguns estudos $^{4-6}$. Giles et al. ${ }^{7}$ afirmam que a gordura corporal total está aumentada e a massa muscular esquelética está diminuída em pacientes portadores de AR quando comparados a controles, com fatores inflamatórios e não inflamatórios contribuindo para essas diferenças.

Os indivíduos que apresentam coexistência de AR e SM estão em risco aumentado para doenças cardiovasculares, que são responsáveis por cerca de metade de todas as mortes em tais pacientes. Este fato é atribuído a um processo acelerado de aterosclerose em virtude da presença de um estado inflamatório sistêmico e a alta prevalência de fatores de risco cardiovasculares tradicionais ${ }^{8}$. A resistência à insulina, considerada um dos componentes-chave da SM, é também comum entre pacientes com AR. Por outro lado, a inflamação crônica também está implicada na patogênese da $\mathrm{SM}^{9,10}$.

Os aumentos nas citocinas pró-inflamatórias, como a interleucina-1 (IL1), interleucina-6 (IL-6), interleucina-18 (IL-18), resistina, fator de necrose tumoral alfa (TNF alfa) e proteína C reativa (PCR), refletem superprodução pela massa de tecido adiposo expandida ${ }^{11}$. Os macrófagos localizados no tecido adiposo parecem ser a fonte primária dessas citocinas e sua ação sistêmica. Entretanto, ainda não está bem esclarecido se a resistência à insulina é causada pelos efeitos parácrinos ou endócrinos dessas citocinas ${ }^{11}$. A adiponectina, uma citocina antiinflamatória produzida apenas por adipócitos, está reduzida na $\mathrm{SM}^{12}$. Sua função consiste em aumentar a sensibilidade à insulina e inibir várias etapas no processo inflamatório, além de inibir a expressão das enzimas relacionadas à gliconeogênese (produção de glicose a partir de substratos metabólicos que não carboidratos) hepática e aumentar a captação de glicose no músculo esquelético ${ }^{12}$.

Níveis aumentados de citocinas pró-inflamatórias oriundas do tecido adiposo estão associadas a AR; a obesidade está associada a um aumento no risco de desenvolver AR, e as citocinas inflamatórias podem desestabilizar uma AR estável ${ }^{13}$.

Em meta-análise recente de quatro estudos transversais controlados e oito estudos de caso-controle envolvendo 2.283 casos e 4.403 controles, Zhang et al. ${ }^{14}$ identificaram uma associação significativa entre AR e o risco de SM (OR:1,24; IC95\%: 1,03-1,50), concluindo que os pacientes com AR têm maior prevalência de SM quando comparados a indivíduos em AR.

Assim, percebe-se a importância de um estudo que objetive esclarecer a relação entre a SM e a AR, visto que ambas as condições constituem fatores de risco para doença aterosclerótica, elevando o número de mortes por doença cardiovascular e ambas podem agir sinergicamente, dificultando o controle da atividade inflamatória da AR. Desta forma, este estudo tem por objetivo identificar a influência da SM na resposta ao tratamento dos pacientes portadores de AR.

\section{Metodologia}

Foi realizado estudo do tipo transversal, descritivo e analítico, no período de setembro de 2013 a junho de 2014, após aprovação do Comitê de Ética em Pesquisa do Centro Universitário do Pará (CESUPA) sob o parecer $n^{\circ} 369.146$. A coleta de dados foi efetuada via protocolo de pesquisa elaborado pelos autores no ambulatório de Reumatologia do Centro de Especialidades Médicas do Centro Universitário do Estado do Pará (CEMEC).

Como condição para participar da pesquisa, os pacientes assinaram o Termo de Consentimento Livre e Esclarecido (TCLE). Foram incluídos na pesquisa os pacientes atendidos no ambulatório de Reumatologia do CEMEC, do sexo feminino, maiores de 18 anos e com diagnóstico de AR segundo os critérios do American College of Rheumatology / European League Against Rheumatism (ACR/EULAR) ${ }^{15}$ em acompanhamento regularmente no CEMEC. Foram excluídos da pesquisa todos os pacientes que não aceitaram assinar o TCLE, tabagistas, associação com outra (s) doença (s) autoimune (s), nefropatia (creatinina $>1,5 \mathrm{mg} / \mathrm{dl}$ ), hepatopatia (AST e/ou ALT > 2 vezes o limite superior da normalidade), bem como mulheres gestantes, menopausadas e/ou em uso de contraceptivos hormonais.

A pesquisa seguiu as normas internacionais de pesquisa em seres humanos. Os pacientes que aceitaram participar da pesquisa responderam ao protocolo de pesquisa, que constava de informações como idade, 
sexo, tempo de doença, peso, altura, circunferência abdominal, pressão arterial sistólica (PAS), pressão arterial diastólica (PAD), glicemia de jejum, perfil lipídico, medicamentos em uso com suas respectivas doses e fator reumatoide.

Para avaliar a atividade inflamatória da doença, foram registrados os valores de velocidade de hemossedimentação (VHS), proteína C reativa (PCR) e calculado o Escore de Atividade da Doença de 28 articulações / Disease Activity Score (DAS28) através dos dados VHS, contagem de articulações doloridas, edemaciadas e avaliação global do paciente ${ }^{16}$.

Após a coleta dos dados, os indivíduos classificados como portadores de SM foram comparados ao grupo de pacientes com AR sem SM, a fim de observar as diferenças entre os grupos, especialmente com relação a atividade inflamatória da doença.

Os dados recolhidos foram organizados e analisados através de planilhas do Microsoft Excel $2010^{\circledR} \mathrm{e}$ do Statistical Package for the Social Sciences (SPSS) 16.0 ${ }^{\circledR}$. As variáveis categóricas foram apresentadas em valores absolutos e percentuais, sendo utilizado o teste do qui-quadrado (com correção de Fisher e razão de verossimilhança quando necessário) para avaliar as diferenças entre os grupos. As variáveis contínuas foram expressas como média \pm desvio-padrão e foram utilizados os testes $t$ de Student e Mann-Whitney para avaliar diferenças estatísticas, sendo utilizado o teste de Kolmogorov Smirnov para avaliação de normalidade dos dados. $\mathrm{O}$ valor de $p<0,05$ foi considerado como estatisticamente significativo.

\section{Resultados}

Foram avaliados 69 pacientes com AR em acompanhamento no ambulatório de Reumatologia do CEMEC, sendo que 27 (39,13\%) pacientes preenchiam os critérios de inclusão e exclusão do estudo, correspondendo a amostra analisada. Na Tabela 1 verificam-se as características clínicas e laboratoriais dos pacientes.

A maioria dos pacientes usava prednisona (92,6\%), com dose média de 9,60 $\pm 7,20 \mathrm{mg} / \mathrm{dia}$. Dentre as drogas modificadoras da doença (DMARD), destaca-se o uso do metotrexato (MTX) por 20 pacientes $(74,1 \%)$, na dose média de 15,70 $\pm 4,69 \mathrm{mg}$ / semana. Dentre os hipolipemiantes, apenas $2(7,4 \%)$ pacientes usavam estatinas (Tabela 2).

Tabela 1. Características clínicas e laboratoriais dos pacientes com AR, CEMEC-CESUPA, Belém-PA, set/2013 - jun/2014

\begin{tabular}{lc}
\hline & $\mathbf{n}=\mathbf{2 7}$ \\
\hline Sexo Feminino n (\%) & $27(100)$ \\
\hline Tempo de doença (anos) & $7,67 \pm 6,02$ \\
\hline Idade (anos) & $40,89 \pm 6,71$ \\
\hline Fator Reumatoide positivo n (\%) & $21(77,8)$ \\
\hline Peso (kg) & $63,71 \pm 11,72$ \\
\hline Altura (cm) & $153,87 \pm 7,86$ \\
\hline Índice de Massa Corpórea (kg/m²) & $26,90 \pm 4,18$ \\
\hline Circunferência Abdominal (cm) & $89,07 \pm 7,95$ \\
\hline PAS (mmHg) & $127,78 \pm 19,28$ \\
\hline PAD (mmHg) & $82,96 \pm 11,03$ \\
\hline Glicemia (mg/dl) & $81,33 \pm 15,37$ \\
\hline Colesterol total (mg/dl) & $197,30 \pm 41,12$ \\
\hline HDL (mg/dl) & $50,05 \pm 14,93$ \\
\hline LDL (mg/dl) & $101,82 \pm 34,95$ \\
\hline Triglicerídeos (mg/dl) & $144,95 \pm 80,46$ \\
\hline VHS (mm/h) & $35,07 \pm 17,43$ \\
\hline PCR (mg/dl) & $13,85 \pm 18,60$ \\
\hline DAS28 & $4,69 \pm 1,18$ \\
\hline
\end{tabular}

Fonte: dados resultantes da pesquisa. 
Dos 27 pacientes, 9 (33,3\%) apresentaram SM. A Tabela 3 apresenta a comparação entre os pacientes com SM e aqueles sem SM. Nota-se que o grupo sem síndrome metabólica apresentou valores menores de pressão sistólica e triglicerídeos e maiores de lipoproteína de alta densidade / high density lipoprotein (HDL), em relação ao grupo com SM.

Na Tabela 4 observa-se a relação entre SM e a atividade da doença de acordo com o DAS28 e as provas de função inflamatória. Não houve diferença estatística de acordo com os parâmetros de atividade avaliados entre os dois grupos.

Entre os pacientes com SM, todos usavam prednisona, com uma dose média de $8,33 \pm 4,33 \mathrm{mg} /$ dia, enquanto que no grupo sem SM, 16 (88,88\%) usavam prednisona, com dose uma média de 10,31 $\pm 8,45 \mathrm{mg} / \mathrm{dia}$. Entre os pacientes com SM, 7 (77,77\%) usavam metotrexato, com uma dose média de $15,71 \pm 4,49 \mathrm{mg} /$ semana, enquanto que no grupo sem SM, 13 (72,22\%) usavam metotrexato, com uma dose média de 15,69 4 4,97 mg/semana. Não houve diferença entre os grupos com relação ao uso de estatina (Tabela 5).

Aplicando-se os diferentes critérios para SM, os critérios da Federação Internacional do Diabetes / International Diabetes Federation (IDF) mostraram-se mais sensíveis, presente em 9 (50\%) dos 18 pacientes que apresentavam SM. Não foi observada associação entre o critério de definição de SM e o nível de atividade de doença, segundo a estratificação do DAS 28 (Tabela 6).

Tabela 2. Distribuição dos portadores com AR de acordo com o uso de medicamentos, CEMEC-CESUPA, Belém-PA, set/2013 - jun/2014

\begin{tabular}{lcc} 
& $\mathbf{n}$ & $\mathbf{\%}$ \\
Prednisona & 25 & 92,6 \\
\hline Metotrexato & 20 & 74,1 \\
\hline Leflunomida & 5 & 18,5 \\
\hline Etanercepte & 1 & 3,7 \\
\hline Estatina & 2 & 7,4 \\
\hline Anti-hipertensivo & 3 & 11,1 \\
\hline
\end{tabular}

Fonte: dados resultantes da pesquisa.

Tabela 3. Características gerais dos pacientes com AR de acordo com a presença de SM, CEMEC-CESUPA, Belém-PA, set/2013 -jun/2014

\begin{tabular}{lccc}
\hline & Com Síndrome Metabólica (n=9) & Sem Síndrome Metabólica (n=18) & $p$-valor \\
Sexo Feminino n (\%) & $9(100)$ & $18(100)$ & - \\
\hline Tempo de doença (anos) & $9,11 \pm 9,00$ & $6,94 \pm 3,93$ & 0,507 \\
\hline Idade (anos) & $44,22 \pm 5,60$ & $39,22 \pm 6,73$ & 0,056 \\
\hline Fator Reumatoide positivo n (\%) & $6(66,66)$ & $15(83,33)$ & 0,367 \\
\hline Peso (kg) & $63,84 \pm 14,64$ & $63,64 \pm 10,46$ & 0,971 \\
\hline Altura (cm) & $152,11 \pm 7,60$ & $154,75 \pm 8,05$ & 0,416 \\
\hline Índice de Massa Corpórea $\left(\mathrm{kg} / \mathrm{m}^{2}\right)$ & $27,52 \pm 4,38$ & $26,59 \pm 4,17$ & 0,606 \\
\hline Circunferência Abdominal (cm) & $90,33 \pm 6,34$ & $88,44 \pm 8,74$ & 0,529 \\
\hline PAS (mmHg) & $147,78 \pm 13,94$ & $117,78 \pm 12,62$ & 70,001 \\
\hline PAD (mmHg) & $90,00 \pm 11,18$ & $79,44 \pm 9,37$ & 0,029 \\
\hline Glicemia (mg/dl) & $83,11 \pm 12,48$ & $80,44 \pm 16,90$ & 0,648 \\
\hline Colesterol total (mg/dl) & $203,67 \pm 35,44$ & $194,11 \pm 44,31$ & 0,551 \\
\hline HDL (mg/dl) & $41,33 \pm 12,31$ & $54,67 \pm 14,38$ & 0,023 \\
\hline LDL (mg/dl) & $102,33 \pm 39,17$ & $101,55 \pm 33,78$ & 0,961 \\
\hline Triglicerídeos (mg/dl) & $201 \pm 103,61$ & $116,92 \pm 48,52$ & 0,044 \\
\hline
\end{tabular}

Fonte: dados resultantes da pesquisa. 
Tabela 4. Relação entre SM e atividade da doença de acordo com o DAS28 e as provas de função inflamatória nos portadores de AR, CEMEC-CESUPA, Belém-PA, set/2013 - jun/2014

\begin{tabular}{lccc}
\hline & $\begin{array}{c}\text { Com Síndrome } \\
\text { Metabólica (n=9) }\end{array}$ & $\begin{array}{c}\text { Sem Síndrome } \\
\text { Metabólica (n=18) }\end{array}$ & p-valor \\
\hline DAS28 & $4,38 \pm 0,74$ & $4,84 \pm 1,34$ & 0,263 \\
\hline VHS $(\mathrm{mm} / \mathrm{h})$ & $33,44 \pm 13,87$ & $35,94 \pm 19,39$ & 0,709 \\
\hline PCR $(\mathrm{mg} / \mathrm{dl})$ & $19,33 \pm 26,66$ & $11,11 \pm 13,07$ & 0,403 \\
\hline
\end{tabular}

Fonte: dados resultantes da pesquisa.

Tabela 5. Distribuição do uso de medicamentos entre os pacientes com SM e sem SM nos portadores de AR, CEMEC-CESUPA, Belém-PA, set/2013 - jun/2014

\begin{tabular}{lccc}
\hline & $\begin{array}{c}\text { Com Síndrome } \\
\text { Metabólica (n=9) }\end{array}$ & $\begin{array}{c}\text { Sem Síndrome } \\
\text { Metabólica (n=18) }\end{array}$ & $p$-valor \\
\hline Prednisona & $9(100 \%)$ & $16(88,88 \%)$ & 0,436 \\
\hline Metotrexato & $7(77,77 \%)$ & $13(72,22 \%)$ & 0,571 \\
\hline Leflunomida & $1(11,11 \%)$ & $4(22,22 \%)$ & 0,447 \\
\hline Etanercepte & $0(0 \%)$ & $1(5,55 \%)$ & 0,654 \\
\hline Estatina & $1(11,11 \%)$ & $1(5,55 \%)$ & 0,564 \\
\hline Anti-hipertensivo & $3(33,33 \%)$ & $0(0 \%)$ & 0,029 \\
\hline
\end{tabular}

Fonte: dados resultantes da pesquisa.

Tabela 6. Distribuição dos portadores de AR de acordo com a atividade da doença e a SM, CEMEC-CESUPA, Belém-PA, set/2013 - jun/2014

\begin{tabular}{|c|c|c|c|c|c|}
\hline \multicolumn{6}{|c|}{ Síndrome Metabólica } \\
\hline \multirow{2}{*}{ Critérios / Atividade da Doença } & \multicolumn{2}{|c|}{ Não } & \multicolumn{2}{|c|}{ Sim } & \multirow{2}{*}{$p$-valor } \\
\hline & $\mathbf{n}$ & $\%$ & $\mathbf{n}$ & $\%$ & \\
\hline \multicolumn{6}{|l|}{ OMS } \\
\hline Remissão & 1 & 3,8 & 0 & 0,0 & \\
\hline Baixa & 2 & 7,7 & 0 & 0,0 & 1,0 \\
\hline Moderada & 14 & 53,8 & 1 & 100,0 & \\
\hline Alta & 9 & 34,6 & 0 & 0,0 & \\
\hline TOTAL & 26 & 100,0 & 1 & 100,0 & \\
\hline \multicolumn{6}{|l|}{ NCEP-ATP III } \\
\hline Remissão & 1 & 4,8 & 0 & 0,0 & \\
\hline Baixa & 2 & 9,5 & 0 & 0,0 & 0,358 \\
\hline Moderada & 10 & 47,6 & 5 & 83,3 & \\
\hline Alta & 8 & 38,1 & 1 & 16,7 & \\
\hline TOTAL & 21 & 100 & 6 & 100,0 & \\
\hline \multicolumn{6}{|l|}{ IDF } \\
\hline Remissão & 1 & 5,6 & 0 & 0,0 & \\
\hline Baixa & 2 & 11,1 & 0 & 0,0 & 0,061 \\
\hline Moderada & 7 & 38,9 & 8 & 88,9 & \\
\hline Alta & 8 & 44,4 & 1 & 11,1 & \\
\hline TOTAL & 18 & 100,0 & 9 & 100,0 & \\
\hline
\end{tabular}

Fonte: dados resultantes da pesquisa. 


\section{Discussão}

Nesta pesquisa, podemos observar que a presença de SM não influenciou na resposta ao tratamento dos pacientes com AR do CEMEC, não guardando relação com a atividade da doença, seja através de exames laboratoriais ou avaliada através do DAS28.

Neste estudo, foi constatada uma frequência de SM igual a 33,3\% (n = 9) nos pacientes com AR, utilizando-se pelo menos um dos critérios estabelecidos para SM, sejam os preconizados pela Organização Mundial da Saúde (OMS), pela National Cholesterol Education Program's Adul Treatmen Panel III (NCEPATP III) ou pela International Diabetes Federation (IDF). O critério que apresentou maior sensibilidade na análise dos pacientes com AR foi o da IDF, através do qual, 9 pacientes foram classificados como portadores de SM, seguido do critério NCEPATP III e da OMS. Esses resultados estão de acordo com o encontrado nos diferentes estudos, que apontam que a frequência de SM varia conforme os critérios diagnósticos empregados ${ }^{14,17,18}$.

Atualmente, várias são as definições existentes para a SM, havendo semelhança entre os componentes centrais da síndrome. Entretanto, alguns valores de corte, bem como a importância de cada componente varia $^{19}$. Nuno Cortez-Dias et al. ${ }^{17}$ afirmam que poucos são os estudos que comparam o valor preditivo para o risco cardiovascular das diferentes definições de SM, e o melhor critério parece estar relacionado com a população estudada. Por exemplo, tal autor, ao correlacionar a presença de doença coronariana com a SM, observou que, de acordo com cada definição empregada, a sensibilidade e a especificidade variaram. A definição da IDF apresentou a melhor sensibilidade $(87,1 \%)$ e menor especificidade $(45,9 \%)$. Já as definições NCEP-ATP III de 2001 e 2004 apresentaram sensibilidade de 82,3 - 84,1\% e especificidade de apenas 48,8 - 50,4\%. De acordo com Brown et al. ${ }^{18}$, análises em populações saudáveis sugerem que a definição da IDF identifica mais homens com SM que o critério NCEP-ATPIII. Paula et al. ${ }^{20}$, comparando os diferentes critérios de definição para diagnóstico de SM em mulheres de 60 a 83 anos, observaram a presença da SM em 30,9\% das pacientes quando em uso dos critérios da NCEP-ATP III, e com o critério da IDF, a SM se fez presente em $45,1 \%$ das idosas.

$\mathrm{Na}$ AR não existe definição sobre qual o critério para SM mais adequado. Aplicando o critério da NCEP-ATP III, Cunha et al. ${ }^{5}$, Karvounaris et al. ${ }^{9}$, Lee et al. ${ }^{21}$, Zonana-Nacah et al. ${ }^{22}$, Raterman et al. ${ }^{23}$, Toms et al. ${ }^{24}$, Dodani et al. ${ }^{25}$ e Salinas et al..$^{26}$ encontraram frequência de SM em pacientes com AR variando de 17 a 40,1\%. Com o critério da OMS, Toms et al. ${ }^{24}$ e Dodani et al. ${ }^{25}$ encontraram frequências iguais a $19,4 \%$ e 13,3\% , respectivamente. Já com o critério da IDF, Karakoc et al. ${ }^{27}$, Toms et al. ${ }^{24}$, Salinas et al. ${ }^{26}$ e Elkan et al. ${ }^{28}$ observaram frequência de SM que variaram de $20 \%$ a $45,3 \%$.

Dao et al. ${ }^{29}$ em estudo transversal no Vietnã, com 105 pacientes com AR e 105 controles, aplicando a definição de SM da NCEP-ATP III e da OMS, observou uma frequência de SM que variou de 16,2\% a 40,9\% em mulheres com AR, e foi maior $(p<0,001)$ do que nos controles $(10,5 \%$ a $22,9 \%)$.

Chung et al. ${ }^{30}$ em estudo com 154 pacientes com AR e 85 controles, encontraram uma frequência de SM igual a $42 \%$ nos pacientes com AR de longa data, $31 \%$ nos pacientes com AR recente e em $11 \%$ nos controles, pelos critérios da OMS ( $\mathrm{p}<0,001)$. Com os critérios do NCEP-ATP III a SM esteve presente em $42 \%$ dos pacientes com AR de longa data, em $30 \%$ dos pacientes com AR recente e em $22 \%$ dos controles $(p=0,03$ ).

Na pesquisa não foi identificada associação estatisticamente significativa entre a presença da SM e a atividade inflamatória na AR. Semelhante aos nossos achados, Sahebari et al. ${ }^{31} \mathrm{em}$ um estudo realizado no Irã sobre a investigação da associação entre SM e a atividade da doença na AR, com uma amostra de 120 pacientes com AR e 500 controles pareados para o sexo e idade, concluíram que a prevalência da SM foi significativamente maior no grupo sem AR. Em relação à atividade da doença, afirma que não foi encontrada qualquer diferença na prevalência da SM entre os pacientes com DAS28 <3,2 e com DAS28 > 3,2, alegando também que não houve associação entre o escore DAS28 e a presença dos componentes da SM segundo as várias definições existentes, além da atividade inflamatória da doença na AR não ser influenciada pela presença da SM. Diferente de nosso trabalho, tal autor utilizou os critérios diagnósticos do ACR de 1987 e apenas os critérios diagnósticos do NCEP-ATP III e da IDF, enquanto que utilizamos os critérios para diagnóstico da AR da ACR/EULAR de 2010 e, também, os critérios pela OMS, NCEP-ATP III e IDF para o diagnóstico de SM.

Karimi et al..$^{32}$, em estudo de caso-controle com 188 mulheres maiores de 18 anos, das quais 92 eram portadoras de AR e 96 eram controles, não encontraram associação estatística significativa entre a presença de SM nos pacientes com AR, aonde, aplicando os critérios do NCEP-ATP III, a frequência da SM nos pacientes com AR foi de 27,2\%, e nos controles, 35,4\% ( $\mathrm{p}=0,22)$. Já com os critérios da OMS, 19,6\% dos 
pacientes com AR e 21,9\% dos controles apresentaram a SM ( $p=0,70)$. Em semelhança com o nosso estudo, tal autor utilizou apenas mulheres maiores de 18 anos.

Lee et al. ${ }^{21}$, realizaram um estudo envolvendo 84 mulheres com AR e 109 controles pareados por idade (faixa de 22 a 76 anos) recrutados de um centro de reumatologia da Coréia do Sul. Utilizaram-se os critérios da ACR de 1987 para diagnóstico de AR e os critérios da NCEP-ATP III usando os critérios asiáticos de obesidade central para o diagnóstico de SM. Também, excluíram de sua amostra pacientes com outras doenças reumáticas, histórico de tomar quaisquer medicamentos, como glicocorticóides ou contraceptivos por via oral pois poderiam alterar a resistência à insulina. No que diz respeito a análise da atividade da doença, utilizaram o DAS28. Esse estudo observou que a frequência de SM não diferiu significativamente entre os pacientes com AR (19\%) e indivíduos saudáveis $(15,6 \%)(\mathrm{p}=0,566)$, embora os pacientes com AR tenham apresentado maior resistência à insulina em comparação com indivíduos saudáveis $(\mathrm{p}<0,001)$.

Por outro lado, autores como da Cunha et al. ${ }^{5}$, Karvounaris et al. ${ }^{9}$, Chung et al. ${ }^{30}$ e Dao et al. ${ }^{29}$, encontraram associação estatística significativa entre a atividade da doença e a presença de SM. No entanto, características dos pacientes estudados e vieses de seleção tornam os seus achados sujeitos a questionamentos.

Em relação à atividade da doença, da Cunha et al. ${ }^{5}$ observaram que o DAS28 foi significativamente maior em pacientes com AR e com SM do que naqueles sem SM (3,59 $\pm 1,27$ vs. $3,14 \pm 1,53, p=0,01)$, resultado que diverge em relação ao nosso estudo. Sabe-se que a idade, tempo de doença e sexo são variáveis que influenciam na história natural desta doença ${ }^{15,33,34}$. Por exemplo, quanto maior o tempo de doença maior a tendência de encontrarmos pacientes em atividade da doença e, também que portadores de AR do sexo masculino tendem a ter melhor prognóstico que os do sexo feminino. Neste contexto, o trabalho desse autor envolveu pacientes com média de idade mais avançada (59,3 anos) e maior tempo de doença (média de 11,1 anos) do que no presente estudo, fato que pode ter influenciado para que encontrasse associação entre atividade da doença e SM.

Chung et al..$^{30}$, em estudo caso-controle com 154 pacientes com AR e 85 controles, aplicando os critérios diagnósticos da OMS e NCEP-ATP III para SM, observaram uma média do DAS28 igual a 3,1 entre os pacientes com AR e SM. Um aspecto importante desse estudo é que os autores analisaram separadamente SM pelo NCEP e pela OMS. Na comparação de atividade entre grupos com e sem SM, nos que tinham critérios da OMS, não houve diferença na atividade da AR. A divergência apenas existiu nos pacientes com o diagnóstico definido pela NCEP-ATP III. Entretanto, este grupo de pacientes apresentava idade (51-59 anos) e tempo de doença maiores (média igual a 20 anos) maiores em relação a nossa amostra, com estas características podendo ter determinado um grupo de pacientes de doença mais ativa e pior prognóstico. Não obstante, o grupo sem SM incluiu mais indivíduos do sexo masculino, de melhor prognóstico, o que pode ter sido importante nos menores níveis de atividade da doença nesta população. Sokka et al. ${ }^{33}$ e Jawaheer et al. ${ }^{34}$ descreveram que homens com AR tendem a ter prognóstico melhor do que mulheres, e com melhor controle da atividade da doença.

Dao et al. ${ }^{29}$, em seu estudo, observaram que a média do DAS28 foi de 4,1. Após ajuste para idade e atividade física, maior taxa de VHS (OR:1,516; IC95\%: 1,073-3,195, p = 0,042), DAS28 (OR:1,736; IC 95\%: $1,293-2,786, p=0,019)$, permaneceram como alguns dos preditores independentes significativos da presença de síndrome metabólica em mulheres com AR. Quanto à atividade da doença, cabe mencionar que o grupo com SM incluiu pacientes com idade (média de 56,3 anos) e tempo de doença maiores (média de 21 meses), além de um maior número de pacientes com fator reumatoide positivo $(73 \%)$, formando então um grupo com tendência a apresentar maior atividade da doença e pior prognóstico. Portanto, a maior atividade da doença nesse grupo pode ter sido determinada por esses fatores de confusão, e não somente pela presença da SM.

Karvounaris et al. ${ }^{9}$ afirmaram que o risco de ter moderada a alta atividade da doença (DAS28 > 3,2) foi significativamente maior em pacientes com síndrome metabólica em comparação com aqueles sem componentes da SM (OR: 9,4; IC95\%: 1,49-57,2, p = 0,016). Quanto à atividade da doença, tal trabalho distribuiu os grupos de acordo com o DAS28 (DAS28 $\leq 3,2$ e $>3,2$ ), no entanto, não relata as características dos dois grupos. Neste caso, pode haver diferença entre os grupos no que diz respeito, por exemplo, ao tempo de doença e positividade do fator reumatoide, características estas que podem ter influenciado o prognóstico dos pacientes. Sem conhecer essas informações, não podemos atribuir a maior atividade da doença a SM, ou simplesmente a um viés. No que diz respeito a variável sexo, pode-se verificar que o grupo sem SM apresentou mais pacientes do sexo masculino (28,57\%) do que o grupo com SM $(23,86 \%)$, podendo ser o fator responsável por amenizar a atividade no grupo sem SM. 
As principais limitações do presente estudo consistiram no tamanho reduzido da amostra, o que impede a generalização e a extrapolação dos resultados. Também, o desenho transversal do estudo, que impossibilita verificar a relação de causalidade, apenas de associação entre as variáveis. Entretanto, como vantagens, temos a homogeneização rigorosa da nossa amostra, o que possibilitou excluir a influência de fatores de confusão importantes, como a idade, o sexo, o tabagismo, presença de outras doenças autoimunes, hepatopatias, nefropatias, gestação, mulheres na menopausa, bem como o uso de contraceptivos hormonais, o que não foi considerado em outros estudos.

\section{Conclusão}

O presente estudo constatou que a presença de SM não influenciou na resposta ao tratamento nos pacientes com AR do CEMEC, não guardando relação com a atividade da doença, seja através de exames laboratoriais ou avaliada através do DAS28, porém, para estabelecer conclusões definitivas e passíveis de generalização, se faz necessária a realização de outros estudos com uma amostra populacional maior e rigorosamente homogênea.

\section{Referências}

1. Santos MJ, Fonseca JE. Metabolic syndrome, inflammation and atherosclerosis - the role of adipokines in health and in systemic inflammatory rheumatic diseases. Acta Reumatol Port. 2009;34(4):590-8. PMid:20852572.

2. Kassi E, Pervanidou P, Kaltsas G, Chrousos G. Metabolic syndrome: definitions and controversies. BMC Med. 2011;9(48):48. http://dx.doi. org/10.1186/1741-7015-9-48. PMid:21542944.

3. Pereira IA, Mota LMH, Cruz BA, Brenol CV, Fronza LSR, Bertolo MB, et al. Consenso 2012 da Sociedade Brasileira de Reumatologia sobre o manejo de comorbidades em pacientes com artrite reumatoide. Rev Bras Reumatol. 2012;52(4):483-95. http://dx.doi.org/10.1590/ S0482-50042012000400002.

4. Crowson CS, Myasoedova E, Davis III JM, Matteson EL, Roger VL, Therneau TM, et al. Increased Prevalence of metabolic syndrome associated with rheumatoid arthritis in patients without clinical cardiovascular disease. J Rheumatol. 2011;38(1):29-35. http://dx.doi.org/10.3899/ jrheum.100346. PMid:20952464.

5. Cunha VR, Brenol CV, Brenol JC, Fuchs SC, Arlindo EM, Melo IM, et al. Metabolic syndrome prevalence is increased in rheumatoid arthritis patients and is associated with disease activity. Scand J Rheumatol. 2012;41(3):186-91. http://dx.doi.org/10.3109/03009742.2011.6264 43. PMid:22416768.

6. Abella V, Scotece M, Conde J, López V, Lazzaro V, Pino J, et al. Adipokines, metabolic syndrome and rheumatic diseases. J Immunol Res. 2014;2014:343746. http://dx.doi.org/10.1155/2014/343746. PMid:24741591.

7. Giles JT, Allison M, Blumenthal RS, Post W, Gelber AC, Petri M, et al. Abdominal adiposity in rheumatoid arthritis: association with cardiometabolic risk factors and disease characteristics. Arthritis Rheum. 2010;62(11):3173-82. http://dx.doi.org/10.1002/art.27629. PMid:20589684.

8. Ferraz-Amaro I, González-Juanatey C, López-Mejias R, Riancho-Zarrabeitia L, González-Gay MA. Metabolic Syndrome in Rheumatoid Arthritis. Mediators Inflamm. 2013;2013:1-11. http://dx.doi.org/10.1155/2013/710928.

9. Karvounaris SA, Sidiropoulos PI, Papadakis JA, Spanakis EK, Bertsias GK, Kritikos HD, et al. Metabolic syndrome is common among middleto-older aged Mediterranean patients with rheumatoid arthritis and correlates with disease activity: a retrospective, cross- sectional, controlled, study. Ann Rheum Dis. 2007;66(1):28-33. http://dx.doi.org/10.1136/ard.2006.053488. PMid:16793841.

10. Zarpellon RSM, Dias MM, Skare TL. Perfil nutricional na artrite reumatoide. Rev Bras Reumatol. 2014;54(1):68-72.http://dx.doi.org/10.1016/j. rbr.2014.01.007. PMid:24878796.

11. Fuentes E, Fuentes F, Vilahur G, Badimon L, Palomo A. Mechanisms of chronic state of inflammation as mediators that link obese adipose tissue and metabolic syndrome. Mediators Inflamm. 2013;2013:136584. http://dx.doi.org/10.1155/2013/136584. PMid:23843680.

12. Cojocaru M, Cojocaru IM, Silosi I, Vrabie CD. Metabolic syndrome in rheumatoid arthritis. Maedica (Buchar). 2012;7(2):148-52. PMid:23399930.

13. Hayashi H, Satoi K, Sato-Mito N, Kaburagi T, Yoshino H, Higaki M, et al. Nutritional status in relation to adipokines and oxidative stress is associated with disease activity in patients with rheumatoid arthritis. Nutrition. 2012;28(11-12):1109-14. http://dx.doi.org/10.1016/j. nut.2012.02.009. PMid:23044162.

14. Zhang J, Fu L, Shi J, Chen X, Li Y, Ma B, et al. The risk of metabolic syndrome in patients with rheumatoid arthritis: a meta-analysis of observational studies. PLoS One. 2013;8(10):e78151. http://dx.doi.org/10.1371/journal.pone.0078151. PMid:24205134. 
15. Aletaha D, Neogi T, Silman AJ, Funovits J, Felson DT, Bingham III CO, et al. 2010 rheumaotid arthritis classification criteria: an american college of rheumatology / european league against rheumatism collaborative initiative. Arthritis Rheum. 2010;62(9):2569-81. http://dx.doi. org/10.1002/art.27584. PMid:20872595.

16. Aletaha D, Smolen J. The Simplified Disease Activity Index (SDAI) and the Clinical Disease Activity Index (CDAI): a review of their usefulness and validity in rheumatoid arthritis. Clin Exp Rheumatol. 2005;23(5 Suppl 39):100-8. PMid:16273793.

17. Cortez-Dias N, Martins S, Belo A, Fiuza M, Investigadores do estudo VALSIM. Comparações de diferentes definições de síndrome metabólica: implicações no risco de doença coronária e acidente vascular cerebral. Rev Port Cardiol. 2011;30(2):139-69. PMid:21553609.

18. Brown TM, Vaidya D, Rogers W, Waters D, Howard B, Tardif J, et al. Does prevalence of the metabolic syndrome in women with coronary artery disease differ by the ATPIII and IDF criteria? J Womens Health (Larchmt). 2008;17(5):841-7. http://dx.doi.org/10.1089/jwh.2007.0536. PMid:18537485.

19. Vinluan CM, Zreikat HH, Levy JR, Cheang KI. Comparison of different metabolic syndrome definitions and risks of incident cardiovascular events in the elderly. Metabolism. 2012;61(3):302-9. http://dx.doi.org/10.1016/j.metabol.2011.07.002. PMid:21840552.

20. Paula HA, Ribeiro RC, Rosado LE, Pereira RS, Franceschini SC. Comparação de diferentes critérios de definição para diagnóstico de síndrome metabólica em idosas. Arq Bras Cardiol. 2010;95(3):346-53. PMid:20676584.

21. Lee SG, Kim JM, Lee SH, Kim KH, Kim JH, Yi JW, et al. Is the frequency of metabolic syndrome higher in South Korean women with rheumatoid arthritis than in healthy subjects? Korean J Intern Med. 2013;28(2):206-15. http://dx.doi.org/10.3904/kjim.2013.28.2.206. PMid:23526131.

22. Zonana-Nacach A, Santana-Sahagún E, Jiménez-Balderas FJ, Camargo-Coronel A. Prevalence and factors associated with metabolic syndrome in patients with rheumatoid arthritis and systemic lúpus erythematosus. J Clin Rheumatol. 2008;14(2):74-7. http://dx.doi.org/10.1097/ RHU.0b013e31816b2faa. PMid:18391674.

23. Raterman HG, van Eijk IC, Voskuyl AE, Peters MJ, Dijkmans BA, van Halm VP, et al. The metabolic syndrome is amplified in hypothyroid rheumatoid arthritis patients: a cross-sectional study. Ann Rheum Dis. 2010;69(1):39-42. http://dx.doi.org/10.1136/ard.2008.100776. PMid:19103637.

24. Toms TE, Panoulas VF, John H, Douglas KM, Kitas GD. Methotrexate therapy associates with reduced prevalence of the metabolic syndrome in rheumatoid arthritis patients over the age of 60 - more than just an anti-inflammatory effect? A cross sectional study. Arthritis Res Ther. 2009;11(4):R110. http://dx.doi.org/10.1186/ar2765. PMid:19607680.

25. Dodani S, Henkhaus R, Wick J, Vacek J, Gupta K, Dong L, et al. Metabolic syndrome in South Asian immigrants: more than low HDL requiring aggressive management. Lipids Health Dis. 2011;10(1):45. http://dx.doi.org/10.1186/1476-511X-10-45. PMid:21410987.

26. Salinas MJ, Bertoli AM, Lema L, Saucedo C, Rosa J, Quintana R, et al. Prevalence and correlates of metabolic syndrome in patients with rheumatoid arthritis in Argentina. J Clin Rheumatol. 2013;19(8):439-43. http://dx.doi.org/10.1097/RHU.0000000000000039. PMid:24263146.

27. Karakoc M, Batmaz I, Sariyildiz MA, Tahtasiz M, Cevik R, Tekbas E, et al. The relationship of metabolic syndrome with disease activity and the funcional status in patients with reumatoid arthritis. J Clin Med Res. 2012;4(4):279-285. PMid:22870176.

28. Elkan AC, Håkansson N, Frostegård J, Cederholm T, Hafström I. Rheumatoid cachexia is associated with dyslipidemia and low levels of atheroprotective natural antibodies against phosphorylcholine but not with dietary fat in patients with rheumatoid arthritis: a crosssectional study. Arthritis Res Ther. 2009;11(2):R37. http://dx.doi.org/10.1186/ar2643. PMid:19284557.

29. Dao H-H, Do Q-T, Sakamoto J. Increased frequency of metabolic syndrome among Vietnamese women with early rheumatoid arthritis: a cross-sectional study. Arthritis Res Ther. 2010;12(6):R218. http://dx.doi.org/10.1186/ar3203. PMid:21182767.

30. Chung CP, Oeser A, Solus JF, Avalos I, Gebretsadik T, Shintani A, et al. Prevalence of the metabolic syndrome is increased in rheumatoid arthritis and is associated with coronary atherosclerosis. Atherosclerosis. 2008;196(2):756-63. http://dx.doi.org/10.1016/j.atherosclerosis.2007.01.004. PMid:17266963.

31. Sahebari M, Goshayeshi L, Mirfeizi Z, Rezaieyazdi Z, Hatef MR, Ghayour-Mobarhan M, et al. Investigation of the association between metabolyc syndrome and disease activity in rheumatoid arthritis. Sci World J. 2011;9(11):1195-205. http://dx.doi.org/10.1100/tsw.2011.111.

32. Karimi M, Mazloomzadeh S, Kafan S, Amirmoghadami $\mathrm{H}$. The frequency of metabolic syndrome in women with rheumatoid arthritis and in controls. Int J Rheum Dis. 2011;14(3):248-54. http://dx.doi.org/10.1111/j.1756-185X.2011.01595.x. PMid:21816020.

33. Sokka T, Toloza S, Cutolo M, Kautiainen H, Makinen H, Gogus F, et al. Women, men, and rheumatoid arthritis: analyses of disease activity, disease characteristics, and treatments in the QUEST-RA Study. Arthritis Res Ther. 2009 jan;11(1):R7. PMid:19144159.

34. Jawaheer D, Messing S, Reed G, Ranganath VK, Kremer JM, Louie JS, et al. Significance of sex in achieving sustained remission in the consortium of rheumatology researchers of north america cohort of rheumatoid arthritis patients. Arthritis Care Res. 2012;64(12):1811-8. http://dx.doi.org/10.1002/acr.21762. PMid:22730408. 


\section{Autor correspondente}

Cezar Augusto Muniz Caldas

Universidade Federal do Pará - UFPA, Instituto de Ciências da Saúde, Internato de Clínica Médica

Avenida Generalíssimo Deodoro, 01, Umarizal

CEP 66050-160, Belém, PA, Brasil

Tel.: (91) 3201-6807

E-mail: cezar_caldas@yahoo.com.br

\section{Informação sobre os autores}

BMF é médico residente de clínica médica do Hospital das Clínicas Gaspar Vianna.

MMRRJ é médico residente de psiquiatria do Hospital das Clínicas Gaspar Vianna.

CAMC é doutor em ciências médicas; médico reumatologista; professor de clínica médica da Universidade Federal do Pará; preceptor do programa de residência médica em reumatologia do Centro Universitário do Estado do Pará (CESUPA)

\section{Contribuição dos autores}

BMF e MMFRJ foram os pesquisadores responsáveis pela revisão literária, coleta e análise de dados; CAMC foi o pesquisador e orientador da pesquisa.

Todos os autores leram e aprovaram a versão final submetida ao Pará Research Medical Journal. 